\title{
Evaluación del ribósido de alopurinol contra la leishmaniasis cutánea ecuatoriana
}

\author{
Martha Chico', Rodrigo Armijos², José Racines², Támara Mancero'1, Ronald H. Guderian ${ }^{3}$
}

\begin{abstract}
Resumen
Se realizó un estudio randomizado de 75 pacientes con leishmaniasis cutánea ecuatoriana con el fin de determinar el índice terapéutico de una nueva droga oral antileishmania, el ribósido de alopurinol más probenecid. Este agente fue evaluado comparándolo con controles positivos que se trataron con pentostam y con testigos no tratados. Las medidas de las lesiones disminuyeron rápidamente durante la terapia, lo que permitió determinar el porcentaje de cicatrización en los diferentes grupos. En el día 70 se realizó la evaluación de la curación de los pacientes en los diferentes grupos, obteniéndose: alopurinol $(1.500 \mathrm{mg} / 6 \mathrm{~h})$ más probenecid $(500 \mathrm{mg} / 6$ h) por 28 días, dió una curación con lesiones cicatrizadas en $10(45,5 \%)$ de los 22 pacientes. Con pentostam ( $20 \mathrm{mg} \mathrm{Sb} / \mathrm{kg} / \mathrm{día}$, IM por 20 días) se obtuvo una curación en 28 pacientes siendo esto el $100 \%$ de cicatrización. El grupo de no tratamiento presentó un porcentaje muy elevado de curación espontánea en $9(75,0 \%)$ de los 12 pacientes. Todos los pacientes con tratamiento fallido o recidivas recibieron posteriormente tratamiento con pentostam.
\end{abstract}

Este estudio sugiere que el ribósido de alopurinol más probenecid no es una droga con mayor eficacia contra la leishmaniasis cutánea ecuatoriana.

\section{Summary}

In a random study of 75 Ecuadorian patients with cutaneous leishmaniasis, the objective was to determine the therapeutical index of a new oral antileishmania drug, allopurinol-riboside (AR) plus probenecid. This agent was evaluated by comparing its effect with positive controls treated with pentostam (Sb) and with other controls who received no treatment at all. The lesions mean area reduced rapidly during treatment, allowing determination of percentage healing in the different groups. The AR group was treated with allopurinal-riboside (1500 mg QID) plus probenecid (500 mg QID) for 28 days. $10(45,5 \%)$ of these 22 patients were found to have healed lesions.

Of those patients treated with Sb (20 mg Sb/kg/día, IM for 20 days), all 28 (100\%) patients presented healed lesions. The control group (that which did not receive any treatment) had an elevated percentage of spontaneous healing in $9(75,0 \%)$ of the 12 patients. Patients who failed to respond to treatment or relapsed, were all later treated with pentostam. This study suggests that allopurinol-riboside plus probenecid has a low efficacy against Ecuadorian cutaneous leishmaniasis.

1 Médica investigadora; Departamento de Investigaciones Clínicas, Hospital Vozandes, Quito, Ecuador.

2 Médico investigador; Departamento de Inmunología, Instituto de Investigaciones, Facultad de Ciencias Médicas, Universidad Central del Ecuador, Quito, Ecuador.

3 Médico Investigador, Director, Departamento de Investigaciones Clínicas, Hospital Vozandes, Quito, Ecuador. 
La leismaniasis cutánea es un grave problema de salud pública en el Ecuador, por su frecuencia. Se presenta como una zoonosis en la mayor parte de la selva húmeda tropical y subtropical de nuestro país. La leismaniasis en el Nuevo Mundo característicamente se presenta como pápulas o úlceras alargadas con una evolución medica de 1-2 meses. Algunas lesiones cicatrizan por sí solas en menos de 2 años. Las lesiones frecuentemente tienen una forma de presentación múltiple. Generalmente, se presentan como una sobreinfección y pueden dar metástasis a los ganglios linfáticos más cercanos y a la orofaringe dando la leishmaniasis mucocutánea. Sin embargo, ésta es una patología que no produce incapacidad, lo que hace que los pacientes no busquen ayuda profesional rápida.

El antimonio pentavalente (Sb), como gluconato de antimonio (Pentostam $₫$ ) o antimoniato de meglumina (Glucantime $₫$ ) (1) han sido utilizados en el tratamiento clásico de la leishmaniasis por muchos años, tiempo en el que se ha demostrado su eficacia en la leishmaniasis cutánea. Aún si la eficacia del $\mathrm{Sb}$ es reconocida, los problemas con esta droga se mantienen: su alto costo; la necesidad de su administración parenteral por algunas semanas, hace que resulte extremadamente difícil utilizarlo en países en desarrollo; la alta toxicidad cardiaca y hepática del fármaco hace que sea necesario realizar exámenes de laboratorio frecuentes que son muy costosos y no es posible hacerlos en los lugares en que se presenta la patología; difícil adquisición y la presencia de algunos casos resistentes a la terapéutica.

Para los tratamientos fallidos con $\mathrm{Sb}$, se pueden utilizar otras drogas antileishmania como la pentamidina o la anfotericina $B$, pero tienen una toxicidad mayor que el $\mathrm{Sb}$ por lo que generalmente no se los utiliza como terapia inicial.

Se han reportado trabajos en los que se evalúa las ventajas de los diferentes tratamientos y casos testigos. Con $\mathrm{Sb}$, una dosis diaria de 20 $\mathrm{mg} / \mathrm{kg} /$ día por 20 días dió una curación del $100 \%$ de pacientes infectados con Leishmania panamensis en Panamá (2); a una dosis de 10$13 \mathrm{mg} \mathrm{Sb} / \mathrm{kg} /$ día por 20 días dió una curación del $76 \%$ de los pacientes $(2,3)$; los casos testigos no curaron espontáneamente (3). Existen estudios con agentes orales antileishmania como los imidazoles y los análogos de las purinas. Los imidazoles, como el Ketoconazol $\circledast$, da una efectividad moderada contra la enfermedad en Centroamérica (Leishmania panamensis) (3), pero es menos efectiva en América del Sur (1).

En el uso de los análogos de las purinas, el alopurinol obtuvo una curación sin recaída en 3 de 6 casos de Kala-azar en la India (4) y de 3 de 6 pacientes con Kala-azar en Kenya, que no habían curado completamente con Sb (5). En otro trabajo en Kenya, una combinación de alopurinol más $\mathrm{Sb}$ fue efectiva en los 5 casos, en quienes fue fallido previamente sólo con $\mathrm{Sb}$ (6). El ribósido de alopurinol se ensayó como agente antileishmania en Panamá, en donde 3 de 9 pacientes presentaron una curación (7). En otros 9 pacientes a quienes se les administraba alopurinol más probenecid, 5 curaron. El estudio de Sáenz y col. sugiere que esta combinación es quizás más efectivo contra la leishmaniasis cutánea americana. En el Ecuador, para determinar la efectividad del alopurinol más probenecid contra la leishmaniasis cutánea ecuatoriana, se relizó un estudio randomizado de 75 pacientes, en el cual se incluyeron controles positivos que fueron tratados con pentostam y testigos que no recibieron tratamiento. Aquí se informan los resultados del estudio.

\section{Materiales y métodos}

Selección de los pacientes: setenta y cinco pacientes ecuatorianos, con lesiones cutáneas clínicamente diagnosticadas de leishmaniasis, se incluyeron en este estudio, previo consentimiento. Para su ingreso se demostró por laboratorio, la presencia del parásito (Leishmania) en las lesiones. Se excluyeron del estudio a aquellos pacientes que presentaban: lesiones mucocutáneas, enfermedades concomitantes graves o que comprometieran la salud del individuo, anormalidad en los exámenes de laboratorio (biometría hemática, VSG, glucosa, 
úrea, creatinina, ácido úrico, bilirrubina total, directa e indirecta, SGOT, SGPT, fosfatasa alcalina, $\mathrm{Na}, \mathrm{K}, \mathrm{Cl}, \mathrm{EMO}$ ), o en las radiografías y ECG que se les realizó previamente a su inclusión.

\section{Diagnóstico}

El diagnóstico clínico se confirmó por el diagnóstico parasitológico mediante: frotis (coloración Giemsa), cultivo (medio de cultivo NNN y de Schneider Drosophila), histopatológico (coloración de hematoxilina-eosina) e inmunofluorescencia directa con anticuerpos monoclonales específicos (DIFMAT8). Las cepas obtenidas de los culticos fueron tipificadas por electroforesis isoenzimática (9).

Todas las muestras de tejidos se obtuvieron del borde activo de las lesiones. Para el frotis se utilizó una hoja de bisturí No. 20 y el material obtenido se fijó en una lámina con metanol para luego colorearla con Giemsa y observar la presencia o no de amastigotes. Para la biopsia, previa anestesia con $1 \mathrm{cc}$ de xilocaína al $2 \%$ con epinefrina, se utilizó un punch de $4 \mathrm{~mm}$ y esta muestra se dividió en tres partes iguales: una para el medio de cultivo NNN, otra para histopatología y la otra para realizar la impronta para inmunofluorescencia. La muestra para histopatología se conservó en solución de Ridley. Se realizaron aspirados utilizando una jeringuilla $3 \mathrm{cc}$ con aguja No. 23 y $0,5 \mathrm{cc}$ de PBS estéril para los medios de cultivo de leishmania: NNN y de Schneider Drosophila; al primero, además, se le añadió parte de la biopsia. Los cultivos se mantuvieron a $25^{\circ} \mathrm{C}$ por 30 días, reportándose entonces como negativo para promastigotes.

\section{Protocolo del estudio}

Los pacientes seleccionados se randomizaron en tres grupos de estudio diferentes: grupo I: 30 pacientes, tratados con ribósido de alopurinol $(1.500 \mathrm{mg} / 6 \mathrm{~h}$, VO) más probenecid (500 mg/6 h, VO) por 28 días; grupo II: 30 pacientes, control positivo, que recibieron pentostam (Sb) $(20 \mathrm{mg} / \mathrm{kg} / \mathrm{día}$ IM por 20 días); y, grupo III: 15 pacientes, grupo testigo sin tratamiento. A los grupos I y II se les realizó una monitoría clínica diariamente durante la terapia, ante la posibilidad de que presentaran algún tipo de reacción adversa; igualmente, se les realizaron controles con exámenes de laboratorio (ya mencionados) en los días 7 y 14 durante el tratamiento y día 70 de seguimiento.

Al día 70 se realizó la evaluación de la respuesta a la terapia en los grupos I y II y para el grupo III la respuesta a su evolución sin tratamiento. El seguimiento posterior para poder evaluar recidivas o reinfecciones para los tres grupos se realizó en los días 90, 180 y 365 . Todas las lesiones se midieron (cm) semanalmente durante el tratamiento y su seguimiento posterior por 365 días. Se asumió que las lesiones eran ovoides y el área de las lesiones se calculó multiplicando la longitud de los dos ejes por el factor 0,78 .

Para clasificar una lesión como curada, era necesaria la presencia de una reepitelización completa y falló si la lesión presentaba menos del $50 \%$ de disminución del área en el día 70 de seguimiento. Al mismo tiempo, se les realizaron cultivos de las lesiones cicatrizadas o no, en busca de parásitos. La lesión se consideraba como recidivante cuando aumentó su longitud después de su cicatrización inicial. La determinación de la respuesta al tratamiento se basó en el criterio clínico; así, a una persona se le define como curada completamente de leishmaniasis si todas las lesiones cicatrizan y no recidivan.

\section{Resultados}

Pacientes: las características de los tres grupos que se incluyeron en este estudio se resumen en la tabla 1 . No hubo una diferencia significativa entre el número de los hombres y mujeres y el promedio de las edades en los tres grupos. En los tres grupos, la mayoría presentaron una lesión única, seguida por los que presentaron dos y tres lesiones. No hubo una diferencia significativa en el tiempo de evolución. La mayoría de las lesiones se localizaban en las extremidades, en los tres grupos. EI promedio del diámetro de las lesiones era menor en el grupo de testigos en comparación a las de los grupos I y II. 
Tabla 1. Características generales de los tres grupos de tratamiento incluidos en el estudio de ribósido de alopurinol.

\begin{tabular}{cccc}
\hline Grupos & \multicolumn{1}{c}{ (RA) $^{\star}$} & II (Sb) & III (NT) $^{\star}$ \\
\hline $\mathrm{n}$ & 30 & 30 & 15 \\
Sexo Hombres & $17(56,6 \%)$ & $16(53,3 \%)$ & $8(53,3 \%)$ \\
Mujeres & $13(43,4 \%)$ & $14(46,7 \%)$ & $7(46,7 \%)$ \\
Edad promedio & 28 & 29 & \multicolumn{2}{c}{34} \\
\hline
\end{tabular}

No. de lesiones

\begin{tabular}{rrrr}
1 & $18(60,0 \%)$ & $16(53,4 \%)$ & $11(73,4 \%)$ \\
2 & $9(30,0 \%)$ & $7(23,3 \%)$ & $2(13,3 \%)$ \\
3 & $3(10,0 \%)$ & $7(23,3 \%)$ & $2(13,3 \%)$ \\
\hline
\end{tabular}

Tiempo de evolución

\begin{tabular}{lrrrr}
$<3$ meses & $15(50,0 \%)$ & $15(50,0 \%)$ & $6(40,0 \%)$ \\
De 3 a 6 meses & $14(46,7 \%)$ & $10(33,3 \%)$ & $7(46,7 \%)$ \\
$>6$ meses & $1(3,3 \%)$ & $5(16,7 \%)$ & $2(13,3 \%)$ \\
\hline Localización & & & & \\
Cabeza & $6(20,0 \%)$ & $8(26,7 \%)$ & $2(13,3 \%)$ \\
Tronco & $3(10,0 \%)$ & $0(0,0 \%)$ & $2(13,3 \%)$ \\
Extremidades & $18(60,0 \%)$ & $18(60,0)$ & $11(73,4 \%)$ \\
Mixta & $3(10,0 \%)$ & $4(13,3 \%)$ & 0 & $(0,0 \%)$ \\
\hline Diámetro pr & $4,4 \mathbf{c m}$ & $\mathbf{3 , 8} \mathbf{~ c m}$ & $\mathbf{1 , 3} \mathbf{~ c m}$ \\
\hline
\end{tabular}

*AR: tratamiento con ribósido de alopurinol

$\mathrm{Sb}$ : tratamiento con pentostam

NT: no tratamiento

Método de diagnóstico: a los 75 pacientes se les realizó la identificación del parásito por cuatro métodos: frotis, cultivo, histopatología e inmunofluorescencia con anticuerpos monoclonales específicos. Los resultados se encuentran en la tabla 2. La inmunofluorescencia con anticuerpos monoclonales era más específica comprada con los otros métodos. En 11 $(14,7 \%)$ pacientes, el diagnóstico se realizó con un solo método (inmunofluorescencia), en 21 $(28,0 \%)$ pacientes dos métodos fueron positivos, en $18(24,0 \%)$ pacientes, tres métodos fueron positivos y en $25(33,3 \%)$ pacientes, los cuatro métodos fueron positivos.

Identificación de los parásitos: en 26 de los 37 casos positivos para cultivo, se obtuvo un número suficiente de promastigotes para lograr su caracterización por medio de electroforesis isoenzimática, tipificándolas a 23 dentro del complejo braziliensis y tres en el complejo mexicana: 12 de Leishmaniasis panamensis, 7 de Leishmania guyanensis, 4 de Leishmania
Tabla 2. Prevalencia de positividad de los cuatro métodos utilizados en el diagnóstico de leishmaniasis.

\begin{tabular}{lcc}
\hline \multicolumn{1}{c}{ Método } & \multicolumn{2}{c}{ Positividad } \\
& No. & (\%) \\
\hline Frotis & 40 & 53,3 \\
Cultivo & 37 & 49,3 \\
Histopatología & 55 & 73,4 \\
DIFMAT & 75 & 100,0 \\
\hline
\end{tabular}

braziliensis, 1 de Leishmania mexicana y 2 de Leishmania amazonensis, las cuales fueron previamente reportadas (10). No hubo una correlación entre las cepas encontradas y la curación clínica y la falla en cada uno de los tres grupos de tratamiento.

\section{Respuesta al tratamiento}

Ribósido de alopurinol: en los pacientes del grupo I tratados con RA más probenecid, la medida promedio de las lesiones pretratamiento $\left(4,4 \mathrm{~cm}^{2}\right)$ fue significativamente mayor que en los pacientes de los grupos I y II. Nueve pacientes curaron bajo el criterio de la disminución de más del $50 \%$ de las lesiones el día 70 de seguimiento. La medida promedio inicial disminuyó en $57 \%, 20 \%$, $9 \%$ y $2 \%$ en los días $7,14,21$ y 28 del tratamiento. Hubo 10 pacientes, quienes presentaron tratamiento fallido, en los que, por lo menos, una lesión cicatrizó menos del $50 \%$ en el día 70 de seguimiento.

Las lesiones en los pacientes con tratamiento fallido eran más pequeñas antes de la terapia (me-dida promedio $=3,4 \mathrm{~cm}^{2}$ ) que las lesiones de los pacientes que curaron (medida promedio $=7,0 \mathrm{~cm}^{2}$ ). De la misma manera, el tiempo de evolución en los pacientes fallidos fue menor (promedio $=2,8 \mathrm{~m}$ ) que en los casos curados (promedio $=3,6 \mathrm{~m}$ ). Todos los casos fallidos fueron luego tratados exitósamente con $\mathrm{Sb}$.

Se presentaron dos pacientes cuyos resultados fueron ambiguos. Estos pacientes fueron técnicamente casos fallidos ya que el día 70 de seguimiento una de las lesiones cicatrizó menos del $50 \%$. Estos pacientes no aceptaron ser tratados con $\mathrm{Sb}$; sin embargo, en el día 90 
de seguimiento las lesiones estuvieron completamente cicatrizadas; en uno de estos pacientes la lesión sufrió un incremento continuo hasta el $161 \%$ en el día 70 de seguimiento y sólo cicatrizó en el día 90. En todos los pacientes que tuvieron un tratamiento exitoso, no se presentó ningún caso de recidiva o reinfección hasta el día 365 de seguimiento.

Pentostam: en el grupo II control positivo, tratados con $\mathrm{Sb}, 28$ de 28 pacientes curaron al día 70 de seguimiento. La medida promedio de las lesiones preterapia fue de $3,8 \mathrm{~cm}^{2}$. La medida promedio inicial disminuyó en un $61 \%$, $23 \%$ y $11 \%$ en los días 7,14 , y 21 de tratamiento, respectivamente. Seis lesiones que originalmente estaban entre 0,1 y $15 \mathrm{~cm}^{2}$ (área promedio $=3,4 \mathrm{~cm}^{2}$ ) aumentaron de $4-200 \%$ en el día 7 de tratamiento. Cuatro lesiones que originalmente estaban entre 0,4 y $5,4 \mathrm{~cm}^{2}$ (área promedio $=1,6 \mathrm{~cm}^{2}$ ) curaron completamente al final de la primera semana de tratamiento. Al final de la tercera semana de tratamiento, sin embargo, sólo 5 lesiones tuvieron más del $50 \%$ de su medida inicial y 29 lesiones $(63,0 \%)$ curaron completamente. En una paciente, su lesión presentó recidiva en el día 90 de seguimiento, luego de haber curado en el día 21 de tratamiento. A los 365 días de seguimiento no se presentaron más casos de recidivas o reinfecciones.

Testigos: en el grupo III, sin tratamiento, a pesar que fueron randomizados con $\mathrm{Sb}$ y RA de acuerdo con un esquema predeterminado, la medida promedio de las lesiones $\left(1,3 \mathrm{~cm}^{2}\right)$ fue significativamente pequeña, comparándola con las medidas de los otros grupos. La medida promedio inicial disminuyó en $37 \%, 41 \%$ y $18 \%$ en el día 7, 14 y 21 de observación sin tratamiento. Nueve de los 12 pacientes $(75,0 \%)$ curaron sus lesiones espontáneamente en el día 70 de observación. Cuatro de los pacientes con curación espontánea pidieron tratamiento a los días 70 y 90 de seguimiento, con lo que fue imposible evaluar futuras recaídas en estos pacientes. Las lesiones en tres de los pacientes sin tratamiento no curaron, siendo tratados posteriormente con $\mathrm{Sb}$. De los pacientes que no recibieron ningún tratamiento, a los 365 días de seguimiento no presentaron recidivas ni reinfecciones.

Seguimiento: de los setenta y cinco pacientes ecuatorianos elegidos para entrar en el estudio, sólo 62 terminaron el protocolo con 365 días de seguimiento ( 22 de 30 pacientes tratados con ribósido de alopurinol (RA); 28 de 30 paceintes tratados con pentostam (Sb) y 12 de 15 pacientes testigos sin tratamiento). A los 62 pacientes que completaron el estudio, se les realizaron estudios parasitológicos (frotis y cultivos) a los 70,180 y 365 días de seguimiento para poder evaluar su curación completa. Todas las lesiones que no cicatrizaron fueron sometidas a repetidos estudios parasitológicos siendo en su mayor parte positivas para Leishmania.

Reacciones adversas: ningún paciente tratado con Sb y RA más probenecid presentó cambios significativos en los valores sanguíneos o urinarios. Catorce pacientes que recibieron $\mathrm{Sb}$ $(50,0 \%)$ reportaron mialgias generalizadas, artargias y cefalea. Cinco pacientes tratados con RA más probenecid $(22,7 \%)$ reportaron cefalea e hiporexia.

\section{Discusión}

El objetivo de este estudio fue determinar el índice terapéutico del ribósido de alopurinol más probenecid contra la leishmaniasis cutánea en Ecuador. Fue un estudio randomizado con controles positivos tratados con pentostam y testigos que no recibieron tratamiento. De los 22 pacientes tratados con RA más probenecid, $10(45,5 \%)$ curaron, $12(54,5 \%)$ fallaron. Los dos $(9,0 \%)$ pacientes que fallaron técnicamente en el día 70 de seguimiento, no quisieron recibir tratamiento y se los encontró curados espontáneamente en el día 90 de seguimiento.

Estos datos son muy importantes ya que nuevamente ilustran la curación espontánea que se puede encontrar en leishmaniasis cutánea en nuestro país. En este grupo de tratamiento, los pacientes que curaron, a los 365 días de seguimiento no presentaron ningún tipo de recidivas, mientras que en el grupo II se presentó un caso. Dado que la tasa de cura- 
ción, al final del estudio (día 365) RA más probenecid $(45,5 \%)$ fue significativamente menor que la del $\mathrm{Sb}(96,4 \%)$ y que el grupo $\sin$ tratamiento $(62,5 \%)$, el régimen experimental no fue eficaz en este estudio. Sin embargo, la alta tasa de curación sin tratamiento hace que la tasa de curación del $96 \%$ obtenida con la terapia estándar de $\mathrm{Sb}$, sea estadísticamente sólo ligeramente más significativa, lo que haría dificultoso demostrar la eficacia de cualquier régimen experimental. Podríamos sugerir que el RA más probenecid sea estudiado en áreas con una tasa de curación natural inferior antes de concluir que el régimen no es útil contra la leishmaniasis cutánea en nuestro país.

En estudios previos (2) y en el trabajo actual, se ha demostrado que las lesiones de pacientes tratados con $\mathrm{Sb}$ y RA que no curaron completamente al final de la terapia, lo hicieron en el día 70 de seguimiento, por lo que se podría considerar éste un tiempo prudente para poder determinar el éxito o el fracaso de la curación de las lesiones. Si se observa que una lesión progresa hacia la cicatrización en este tiempo, no debería iniciarse una nueva terapéutica. El fracaso se definió como menos del $50 \%$ de cicatrización en el primer seguimiento. No hay reportes previos sobre medidas semanales de las lesiones en grandes series de pacientes con leshmaniasis cutánea. Este reporte es instructivo porque la información sugiere que la tasa media de reepitelización progresiva sería la esperada. Los datos también indican que ni el aumento de la lesión al inicio de la terapia, ni las lesiones no cicatrizadas al final de la terapia significan fracaso terapéutico.

En este estudio es importante tomar en cuenta que a pesar de que el tamaño de las lesiones en el grupo sin tratamiento antes de la terapia fue significativamente más pequeño que en los otros dos grupos de tratamiento; sin embargo, el tamaño de las lesiones del grupo III destinadas a curar fue virtualmente igual al tamaño de las lesiones destinadas a fracasar, al igual que en el grupo I (RA) en donde las lesiones más pequeñas fueron más propensas a fracasar.
Como se menciona previamente (1), la OMS recomienda que los pacientes con leishmaniasis cutánea deben ser tratados "hasta que la lesión cicatrice", constituyendo un sobretratamiento considerable y más bien las lesiones deberían ser tratadas con una dosis diaria de $\mathrm{Sb}$ por un período predeterninado de días, que ya han sido determinados en estudios previos y que dan una cicatrización de las lesiones en el día 70 de seguimiento. La incidencia muy alta de cura sin tratamiento demuestra extraordinariamente la impotancia de incluir en grupos randomizados un grupo de control sin tratamiento en cualquier investigación terapéutica de leishmaniasis cutánea.

Por los resultados obtenidos en este estudio, el ribósido de alopurinol es una droga con una efectividad muy baja contra la leishmaniasis cutánea en nuestro país. Se debe seguir considerando al pentostam como la droga de elección en esta patología.

\section{Agradecimientos}

Agradecemos al doctor Dimitri Barreto, Decano de la Facultad de Ciencias Médicas de Quito y al doctor Marco Herdoiza, Director del Departamento de Inmunología, Instituto de Investigaciones, de la Universidad Central del Ecuador, Quito, por su estímulo en la realización del proyecto. Al doctor Calos Burneo, doctor Ricardo Izurieta y al doctor Mario Pancho por su participación activa en el trabjo de campo. El financiamiento para este proyecto fue dado por Burroughs Wellcome Co. y Dember Foundation, Inc.

\section{Referencias}

1. Berman JD. Chemotherapy for leishmaniasis: biochemical mechanisms, clinical efficacy and future strategies. Rev Infect Dis 1988;10:560.

2. Ballou WR, McClain JB, Gordon MD, Shanks GD, Andujar J, Berman JD, et al. Safety and efficacy of high dose sodium stibogluconate therapy of American cutaneous leishmaniasis. Lancet 1987;2:3.

3. Sáenz RE, Paz H, Berman JD. Efficacy of ketoconazole against Leishmania braziliensis panamensis cutaneous leishmaniasis. Am J Med 1990;89:147.

4. Jha TK. Evaluation of allopurinol in the treatment of kala-azar occuring in North Bihar, India. Trans R Soc Trop Med Hyg 1983;77:204. 
5. Kager PA, Rees PH, Wellde BT, Hockmeyer WT, Lyerly WH. Allopurinol in the treatment of visceral leishmaniasis. Trans R Soc Trop Med Hyg 1981;75:556.

6. Chunge CN, Gachihi G, Muigai R, Wasuna K, Rashid JR, Chulay JD, et al. Visceral leismaniasis unresponsive to antimonial drug. III Successful treatment using a combination of sodium stibogluconate plus allopurinol. Trans R Soc Trop Med Hyg 1985;79:715.

7. Sáenz RE, Paz HM, Johnson CM, Marr JJ, Nelson DJ, Pattishall KH, et al. Treatment of american cutaneous leismaniasis with orally administered allopurinol riboside. J Infect Dis 1989;160:153.
8. Grogl M, Milhous WK, Martin RK, Kyle DE. Kits for the diagnosis of cutaneous leismaniasis in field laboratories. Proc Soc Armed Forces Med Lab Science 1989;18:22.

9. Kreutzer RD, Souraty N, Semko ME. Biochemical identities and differences among Leishmania species and subspecies. Am J Trop Med Hyg 1987;36:22.

10. Armijos RX, Chico ME, Cruz ME, Guderian RH, Kreutzer RD, Berman JD, et al. Human cutaneous leishmaniasis in Ecuador: identification of parasites by enzyme electrophoresis. Am J Trop Med Hyg 1990;42:424. 